\title{
Comparing Techniques for Mesenteric Defects Closure in Laparoscopic Gastric Bypass Surgery-a Register-Based Cohort Study
}

\author{
Erik Stenberg $^{1}$ (D) $\cdot$ Johan Ottosson $^{1} \cdot$ Eva Szabo $^{1} \cdot$ Ingmar Näslund $^{1}$
}

Published online: 23 January 2019

(C) The Author(s) 2019

\begin{abstract}
Background Routine closure of mesenteric defects is generally considered standard part of laparoscopic gastric bypass surgery today. Controversy still exists regarding the optimal method for mesenteric defects closure. The objective was to compare different methods for mesenteric defects handling in laparoscopic gastric bypass surgery.

Methods Primary laparoscopic gastric bypass procedures from 2010 until 2015 reported to the Scandinavian Obesity Surgery Registry (SOReg), where the mesenteric defects closure method was identifiable, were included. Main outcome measures were serious postoperative complication within 30 days after surgery, and reoperation for small bowel obstruction within 5 years after surgery. Quality-of-life before and after surgery, duration of surgery, and risk factors for complication were also analyzed. Information on operation for small bowel obstruction was based on data from the SOReg, the Swedish National Patient Register and reviews of hospital charts.

Results In all, 34,707 patients were included. Serious postoperative complication occurred in 174 (2.9\%) patients with sutures, in $592(3.1 \%$, adjusted $p=0.079)$ with clips, and $278(3.1 \%$; adjusted $p=0.658)$ in the non-closure group. Reoperation for small bowel obstruction within 5 years after surgery was lower with sutures (cumulative incidence 6.9\%) and clips (cumulative incidence 7.3\%; adjusted HR 1.16, 95\% CI 1.02-1.32, $p=0.026$ ), compared to non-closure (cumulative incidence 11.2\%; adjusted HR $1.63,95 \%$ CI $1.44-1.84, p<0.0001$ ).

Conclusion Closure of the mesenteric defects using either non-absorbable metal clips or non-absorbable running sutures is a safe and effective measure to reduce the risk for small bowel obstruction after laparoscopic gastric bypass surgery. Sutures appear slightly more effective and should remain gold standard for mesenteric defects closure.
\end{abstract}

Keywords Gastric bypass $\cdot$ Bariatric surgery $\cdot$ Internal hernia $\cdot$ Small bowel obstruction $\cdot$ Postoperative complication $\cdot$ Prevention

\section{Background}

The excellent long-term results of gastric bypass surgery [1] together with the low risk for postoperative complication using the laparoscopic technique $[2,3]$ has made way for

Erik Stenberg

erik.stenberg@regionorebrolan.se

Johan Ottosson

johan.ottosson@regionorebrolan.se

Eva Szabo

eva.szabo@ regionorebrolan.se

Ingmar Näslund

ingmar.naslund@regionorebrolan.se

1 Department of Surgery, Faculty of Medicine and Health, Örebro University, SE-70185 Örebro, Sweden the enormous expansion of laparoscopic gastric bypass surgery over the last two decades [4]. During the same period of time, small bowel obstruction due to internal hernia following surgery has increased dramatically [5] with potentially devastating consequences for the patient $[6,7]$. Closure of the mesenteric defects reduces the risk for small bowel obstruction [8] and is now an accepted part of any laparoscopic gastric bypass procedure in many parts of the world. Several methods are available for mesenteric defects closure. Nonabsorbable sutures have the highest tensile strength [9] and best scientific documentation [10], but surgeons are sometimes discouraged by the technical challenge of the technique and the risk for kinking of the jejunojejunostomy resulting in small bowel obstruction in the early postoperative phase [10]. A technique using metal clips is potentially easier to learn and master, and is gaining increasing popularity. To date, no large trial comparing these two methods has been conducted. 
The aim of the present study was to compare closure of the mesenteric defects with sutures, metal clips or nonclosure, based on the hypothesis that there is no difference between sutures and clips, and that the risk for small bowel obstruction is reduced with both methods compared to non-closure.

\section{Methods}

The Scandinavian Obesity Surgery Registry (SOReg) is a national quality and research registry covering virtually all bariatric surgical procedures in Sweden from 2010 onwards [11]. From the SOReg database, all patients operated with a primary laparoscopic gastric bypass procedure between January 1, 2010 and December 31, 2015 were assessed for eligibility. Retrocolic gastric bypass procedures, unclear or mixed methods for mesenteric defects closure, closure of only one mesenteric defect, or conversion to open surgery were considered exclusion criteria. Baseline characteristics, early postoperative complication and quality-of-life were based on SOReg data. Data on operations for small bowel obstruction were based on data from the SOReg, and the Swedish National Patient Register (NPR). Data on reoperations were available from the NPR until December 31, 2015. A diagnosis of small bowel obstruction (ICD-10 codes K45.0, K45.1, K56, and K91.3) with a related, relevant abdominal operation was considered surgery for small bowel obstruction. All charts for patients with a diagnosis of K45.8 (other specified abdominal hernia without obstruction) or with any uncertainty in the registration in SOReg or NPR were reviewed. Small bowel obstruction was then defined as acute presentation of abdominal pain with objective finding of bowel obstruction at surgery $[8,10]$.

\section{Outcome}

Main outcomes were serious complication within 30 days after surgery, and operation for small bowel obstruction within 5 years after surgery. Secondary outcomes were duration of surgery and quality-of-life.

\section{Procedures}

The technique for laparoscopic gastric bypass in Sweden is well standardized, using an antecolic, antegastric laparoscopic gastric bypass procedure [12]. The techniques for mesenteric defects closure with sutures (videos available at: https://s $3 \mathrm{~m}$. io/yZTGe and https://s3m.io/RcFQy) [10], and clips [13] have been described previously.

\section{Definitions}

The Clavien-Dindo classification [14] was used to classify postoperative complications. Complications scoring $\geq 3 \mathrm{~b}$ on the Clavien-Dindo scale (i.e., complications requiring intervention under general anesthesia, resulting in organ failure or mortality) were considered serious postoperative complications.

Quality-of-life at baseline and 2 years after surgery was estimated using the obesity problem scale (OP) and the bodily pain dimension from the short form-36 (SF36RAND). OP is a disease-specific scale measuring the impact of obesity on psychosocial functioning, reporting aggregate scores from 0 to 100 , with lower scores representing better well being [15]. The bodily pain dimension on the SF-36RAND is also reported as a score from 0 to 100 , with higher scoring representing better well being [16]. Comorbidity was defined as a specific obesity-related condition requiring pharmacological treatment or continuous positive airway pressure treatment.

\section{Statistics}

Mesenteric defects closure with sutures was considered reference method. Continuous variables were analyzed using linear regression, categorical variables with logistic regression. For serious postoperative complication, odds ratios (OR) with $95 \%$ confidence intervals $(95 \%$ $\mathrm{CI})$ were reported as measures of association. The risk for reoperation due to small bowel obstruction within 5 years after surgery was estimated and visualized using the Kaplan-Meier method and presented as cumulative probability (1-Kaplan-Meier estimate). All patients were followed until first reoperation due to small bowel obstruction, and censored if the mesenteric defects were closed during any operation, at last known follow-up, at 5 years or December 31, 2015. Cox regression was used to calculate Hazard ratios (HR) with $95 \%$ CI for this outcome. Odds ratios and Hazard ratios were analyzed unadjusted and adjusted for year of surgery, age, sex, body mass index (BMI), and specified comorbidities. Abdominal operations for any cause were estimated using the Kaplan-Meier method and unadjusted Cox regression. Quality-of-life was analyzed as difference between baseline value and reported estimate at 2 years using linear regression unadjusted (adjusted for baseline value), and adjusted for baseline value, age, sex, percentage excess BMI-loss at 2 years after surgery, and specified comorbidities.

$P<0.05$ was considered statistically significant. All data were analyzed using SPSS Statistics version 22. 


\section{Results}

A total of 38,078 patients operated with a primary laparoscopic gastric bypass procedure during the inclusion period were identified. After exclusion of retrocolic procedures $(n=184)$, mixed or non-reported mesenteric defects closure techniques $(n=2294)$, closure of only one mesenteric defect $(n=803)$, and conversion to open surgery $(n=90), 34,707$ patients remained for analysis. Completed quality-of-life forms were collected both at baseline and 2-year follow-up from 9219 patients concerning the OP-scale, and 9190 patients concerning SF-36RAND. Follow-up at day 30 was $98.1 \%$ $(n=34,040)$. The mesenteric defects were closed with nonabsorbable, running sutures in 6149 patients $(17.7 \%)$, with non-absorbable metal clips in 19,436 patients $(56.0 \%)$, and left open in 9122 patients $(26.3 \%)$.

The mean age at the time of surgery was $40.8 \pm 11.24$ years, mean BMI $42.1 \pm 5.27 \mathrm{~kg} / \mathrm{m}^{2}$, the majority were women $(n=$ 26,$284 ; 75.7 \%$ ), and 19,067 (54.9\%) had a comorbid disease (Table 1).

The mean operation time was $70.3 \pm 24.53 \mathrm{~min}$ in the sutures group, $60.6 \pm 20.02 \mathrm{~min}(p<0.0001)$ in the clips group, and $79.9 \pm 36.26 \mathrm{~min}(p<0.0001)$ in the non-closure group. A postoperative complication occurred within 30 days after 2632 (7.7\%) operations, with $1044(3.1 \%)$ meeting the criteria for serious postoperative complication (Table 2). A serious postoperative complication occurred in 174 (2.9\%) patients with sutures, 592 (3.1\%; adjusted OR 1.18, 95\% CI 0.98$1.42, p=0.079)$ with clips, and 278 (3.1\%; adjusted OR $1.05,95 \%$ CI $0.85-1.29, p=0.658$ ) in the non-closure group (Table 3).

A total of 2243 patients underwent reoperation for small bowel obstruction (Fig. 1, Table 4). In the sutures group, 341 patients were operated for small bowel obstruction within 5 years after surgery (cumulative incidence $6.9 \%$ ), while 956 were operated for small bowel obstruction in the clips group (cumulative incidence $7.3 \%$, unadjusted HR $1.13,95 \%$ CI $0.99-1.28, p=0.060$; adjusted HR $1.16,95 \%$ CI $1.02-1.32$, $p=0.0 .026$ ), and 946 in the non-closure group (cumulative incidence $11.2 \%$, unadjusted HR $1.63,95 \%$ CI $1.44-1.84$, $p<0.0001$; adjusted HR $1.7495 \%$ CI 1.53-1.98, $p<0.0001)$. Other significant risk factors were female sex (adjusted HR 1.20, 95\% CI 1.07-1.34, $p=0.001$ ), while increasing age (adjusted HR 0.98, 95\% CI 0.98-0.99/year, $p<0.0001$ ), increased BMI (adjusted HR 0.97, 95\% CI 0.96-0.98/BMI-unit, $p<0.0001$ ), hypertension (adjusted HR $0.80,95 \%$ CI $0.71-0.91, p=0.001$ ), and diabetes (adjusted HR $0.70,95 \%$ CI $0.60-0.83, p<0.0001$ ) were associated with reduced risk.

In the sutures group, 598 patients underwent an abdominal operation within 5 years (cumulative incidence 12.0\%), while 1720 were operated in the clips group (cumulative incidence $12.6 \%, p=0.008$ ), and 1489 in the non-closure group (cumulative incidence $17.4 \%, p<0.0001)$.

\section{Quality-of-Life comparison}

The OP-score prior to surgery was $62.3 \pm 25.40$ in the sutures group, $61.5 \pm 26.35$ in the clips group $(p=0.270)$, and $60.7 \pm$ $26.74(p=0.026)$ in the non-closure group. Two years after surgery, the OP-score was $18.2 \pm 22.63$ in the sutures group, $20.6 \pm 24.27(p<0.0001)$ in the clips group, and $21.7 \pm 24.55$ $(p<0.0001)$ in the non-closure group. The mean improvement in OP-score was lower for clips (mean difference 2.63, 95\% CI 1.46-3.81, $p<0.0001$; adjusted mean difference 2.32, $95 \%$ CI $1.16-3.49, p<0.0001$ ), and non-closure (mean

Table 1 Baseline characteristics

\begin{tabular}{|c|c|c|c|c|c|c|}
\hline & \multicolumn{2}{|l|}{ Sutures } & \multicolumn{2}{|l|}{ Clips } & \multicolumn{2}{|l|}{ No-closure } \\
\hline & Missing data & & Missing data & & Missing data & \\
\hline $\mathrm{BMI}$, mean $\pm \mathrm{SD}, \mathrm{kg} / \mathrm{m}^{2}$ & 0 & $41.1 \pm 5.10$ & 0 & $42.2 \pm 5.29$ & 0 & $42.6 \pm 5.24$ \\
\hline Age, mean $\pm \mathrm{SD}$, years & 0 & $40.9 \pm 11.09$ & 0 & $40.6 \pm 11.33$ & 0 & $41.1 \pm 11.12$ \\
\hline \multicolumn{7}{|l|}{ Sex } \\
\hline Female & 0 & $4801(78.1 \%)$ & 0 & $14,580(75.0 \%)$ & 0 & $6903(75.7 \%)$ \\
\hline Male & 0 & $1348(21.9 \%)$ & 0 & $4856(25.0 \%)$ & 0 & $2219(24.3 \%)$ \\
\hline Comorbidity, $n(\%)$ & 0 & $2991(48.6 \%)$ & 0 & $11,548(59.4 \%)$ & 0 & $4528(49.6 \%)$ \\
\hline Sleep apnea, $n(\%)$ & 0 & $592(9.6 \%)$ & 0 & $2183(11.2 \%)$ & 0 & $782(8.6 \%)$ \\
\hline Hypertension, $n(\%)$ & 0 & $1439(23.4 \%)$ & 0 & $5093(26.2 \%)$ & 0 & $2436(26.7 \%)$ \\
\hline Diabetes, $n(\%)$ & 0 & $669(10.9 \%)$ & 0 & $2959(15.2 \%)$ & 0 & $1328(14.6 \%)$ \\
\hline Dyslipidemia, $n(\%)$ & 0 & $528(8.6 \%)$ & 0 & $1986(10.2 \%)$ & 0 & $980(10.7 \%)$ \\
\hline Dyspepsia/GERD, $n(\%)$ & 0 & $551(9.0 \%)$ & 0 & $2418(12.4 \%)$ & 0 & $712(7.8 \%)$ \\
\hline Depression, $n(\%)$ & 0 & $846(13.8 \%)$ & 0 & $3103(16.0 \%)$ & 0 & $1311(14.4 \%)$ \\
\hline Previous DVT/VTE & $472(7.7 \%)$ & $153(2.7 \%)$ & $303(1.6 \%)$ & $494(2.6 \%)$ & $1573(17.2 \%)$ & $184(2.4 \%)$ \\
\hline
\end{tabular}


Table 2 Specified postoperative complications during day 0-30

\begin{tabular}{|c|c|c|c|c|c|}
\hline & \multirow{2}{*}{$\begin{array}{l}\text { Suture } \\
n(\%)\end{array}$} & \multicolumn{2}{|l|}{ Clips } & \multicolumn{2}{|l|}{ Non-closure } \\
\hline & & $n(\%)$ & $P^{1}$ & $n(\%)$ & $P^{1}$ \\
\hline Any postoperative complication & $478(7.8 \%)$ & $1433(7.6 \%)$ & 0.504 & $721(8.0 \%)$ & 0.812 \\
\hline Leak/intra-abdominal abscess & $66(1.1 \%)$ & $258(1.4 \%)$ & 0.091 & $151(1.7 \%)$ & 0.003 \\
\hline Small bowel obstruction/paralysis & $75(1.2 \%)$ & $225(1.2 \%)$ & 0.802 & $82(0.9 \%)$ & 0.052 \\
\hline Bleeding & $115(1.6 \%)$ & $343(1.8 \%)$ & 0.371 & $194(2.1 \%)$ & 0.030 \\
\hline Other wound complication & $46(0.8 \%)$ & $114(0.6 \%)$ & 0.198 & $75(0.8 \%)$ & 0.625 \\
\hline Port-related complication & $16(0.3 \%)$ & $62(0.3 \%)$ & 0.426 & $50(0.5 \%)$ & 0.010 \\
\hline Stricture & $10(0.2 \%)$ & $41(0.2 \%)$ & 0.428 & $17(0.2 \%)$ & 0.738 \\
\hline Marginal ulcer & $28(0.5 \%)$ & $85(0.5 \%)$ & 0.922 & $39(0.4 \%)$ & 0.789 \\
\hline Cardiovascular complication & $10(0.2 \%)$ & $19(0.1 \%)$ & 0.210 & $19(0.2 \%)$ & 0.531 \\
\hline Pulmonary complication & $50(0.8 \%)$ & $78(0.4 \%)$ & 0.0001 & $63(0.7 \%)$ & 0.378 \\
\hline DVT/VTE & $8(0.1 \%)$ & $16(0.1 \%)$ & 0.311 & $11(0.1 \%)$ & 0.865 \\
\hline Urinary tract infection & $32(0.5 \%)$ & $52(0.3 \%)$ & 0.064 & $42(0.5 \%)$ & 0.592 \\
\hline
\end{tabular}

${ }^{1}$ Based on unadjusted logistic regression using suture group as reference

difference 1.97, 95\% CI 1.36-2.58, $p<0.0001$, adjusted mean difference $1.54,95 \%$ CI $0.93-2.14, p<0.0001)$ compared to sutures.

Bodily pain on the SF-36 RAND prior to surgery was 55.8 \pm 26.94 in the sutures group, $54.5 \pm 27.11(p=0.065)$ in the clips group, and $56.2 \pm 27.06(p=0.599)$ in the non-closure group. Two years after surgery, bodily pain was $75.5 \pm 28.62$ in the sutures group, $72.3 \pm 29.37(p<0.0001)$ in the clips group and $72.3 \pm 29.09(p=0.0001)$ in the non-closure group. The mean improvement in bodily pain was lower for clips (mean difference 2.54, 95\% CI 1.17-3.91, $p=0.0003$, adjusted mean difference $1.94,95 \%$ CI $0.56-3.31, p=0.006)$, and non-closure (mean difference 1.64, 95\% CI 0.93-2.36, $p<0.0001$; adjusted mean difference 1.21 , 95\% CI $0.49-$ $1.92, p=0.001)$ compared to sutures.

Table 3 Severity of postoperative complications during day $0-30$

\begin{tabular}{lccc}
\hline & $\begin{array}{l}\text { Suture } \\
n(\%)\end{array}$ & $\begin{array}{l}\text { Clips } \\
n(\%)\end{array}$ & $\begin{array}{l}\text { Non-closure } \\
n(\%)\end{array}$ \\
\hline No complication & $5612(92.2 \%)$ & $17,454(92.4 \%)$ & $8342(92.0 \%)$ \\
I & $77(1.3 \%)$ & $223(1.2 \%)$ & $90(1.0 \%)$ \\
II & $206(3.4 \%)$ & $545(2.8 \%)$ & $273(3.0 \%)$ \\
IIIa & $21(0.3 \%)$ & $73(0.4 \%)$ & $80(0.9 \%)$ \\
IIIb & $158(2.6 \%)$ & $554(2.9 \%)$ & $258(2.8 \%)$ \\
IV & $11(0.2 \%)$ & $35(0.2 \%)$ & $18(0.2 \%)$ \\
V & $5(0.1 \%)$ & $3(0.0 \%)$ & $2(0.0 \%)$ \\
\hline
\end{tabular}

Severity of postoperative complications classified according to the Clavien-Dindo scale (grade I is a complication without need for pharmacological, surgical, endoscopic, or radiological intervention; grade II requires pharmacological treatment; grade IIIa requires intervention without general anesthesia; grade IIIb intervention under general anesthesia; grade IV single or multi-organ failure; grade V death)

\section{Discussion}

Closure of the mesenteric defects using either sutures or clips in antecolic laparoscopic gastric bypass surgery was associated with lower risk for small bowel obstruction compared to non-closure. However, sutures were slightly more effective after adjustment for other potential risk factors. Both groups improved health-related quality-of-life and bodily pain significantly, but slightly less so with clips compared to sutures. Since there is a strong association between bodily pain and chronic abdominal pain, one possible concern may be that the lower tensile strength of clips may result in slightly higher rates of intermittent internal herniation $[9,17]$. Although the differences in health-related quality-of-life were negligible from a clinical perspective, all things considered, sutures appears slightly more effective than clips.

The main concern with mesenteric defects closure has been related to safety issues, mainly in terms kinking of the jejunojejunostomy $[10,18]$. Several adjustments in technique have been tried in an attempt to reduce this risk, such as an antiobstructive stitch [19], wide division of the mesentery [20], and creation of a wide jejunojejunostomy using a double stapling technique [21]. The scientific support for any of these preventive measures, however, remains weak. Closure of the mesenteric defects using metal clips is generally viewed as a potentially easier technique to master, and may also lead to a slight reduction in the duration of surgery. On the other hand, the use of clips increases instrument costs, which may matter in situations where resources are limited [22]. In the present study, both techniques for mesenteric defects closure were found to be safe, with a tendency for a small, but acceptable, increased risk for bowel obstruction during the first 30 days, compared to non-closure. Both methods were associated with 
Fig. 1 Cumulative probability of operation for small bowel obstruction

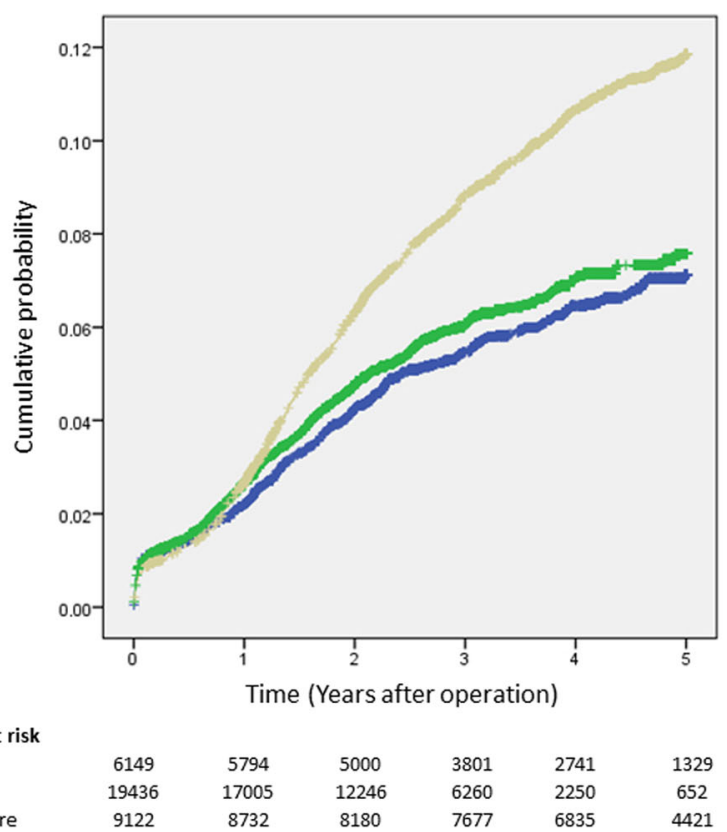

$\begin{array}{cccccc}6149 & 5794 & 5000 & 3801 & 2741 & 1329 \\ 19436 & 17005 & 12246 & 6260 & 2250 & 652 \\ 9122 & 8732 & 8180 & 7677 & 6835 & 4421\end{array}$

Number at ris Suture Clips Non-closure
Suture
Clips
Non-closure been fully established as a risk factor for small bowel obstruction after laparoscopic gastric bypass surgery, the risk for small bowel obstruction is high during pregnancy in this group of patients [29, 30]. Apart from handling of the mesenteric defects, none of these risk factors are preventable. An increased awareness, however, may aid in the evaluation of patients with abdominal pain after laparoscopic gastric bypass surgery.

The study is not without limitations. First, it was a nonrandomized trial. The technique for mesenteric defects closure may therefore be less well controlled. Furthermore, small differences between the study groups may exist. Technically, suturing is generally considered a more challenging technique than using clips. For this reason, performance of this technique may be biased towards more experienced surgeons. On the other hand, surgeons may have chosen sutures to close more technically challenging defects. Since this was an observational study, we do not have information on intention-to-treat. A randomized clinical trial would be the optimal design to compare clips to sutures, but such a trial would be much larger than what was considered possible at the time when this study was designed. In the present study, data from two reliable nationwide

Table 4 Cumulative incidence of reoperation for small bowel obstruction after surgery

\begin{tabular}{llllll}
\hline & $\begin{array}{l}1 \text { year } \\
n(\%)\end{array}$ & $\begin{array}{l}2 \text { years } \\
n(\%)\end{array}$ & $\begin{array}{l}3 \text { years } \\
n(\%)\end{array}$ & $\begin{array}{l}4 \text { years } \\
n(\%)\end{array}$ & $\begin{array}{l}5 \text { years } \\
n(\%)\end{array}$ \\
\hline Suture & $133(2.2 \%)$ & $241(4.2 \%)$ & $295(5.3 \%)$ & $328(6.3 \%)$ & $341(6.9 \%)$ \\
Clips & $493(2.6 \%)$ & $790(4.6 \%)$ & $911(5.9 \%)$ & $947(6.7 \%)$ & $956(7.3 \%)$ \\
Non-closure & $238(2.6 \%)$ & $546(6.1 \%)$ & $746(8.5 \%)$ & $879(10.1 \%)$ & $946(11.2 \%)$ \\
\hline
\end{tabular}

Total number of patients with reoperation for small bowel obstruction at each time with cumulative incidence as estimated by use of the Kaplan-Meier method 
registers complemented by data from hospital charts were used. Despite this, the risk for slight underestimation of the number of reoperations for small bowel obstruction remains. Based on previous reports, it is likely that this would affect the control group to a greater extent and would therefore underestimate the beneficial effect of treatment of mesenteric defects closure to some extent [8]. Many patients in the clip group were censored before postoperative year five due to limited follow-up time, limiting the evaluation of long-term efficacy for this group. There is therefore a risk that higher long-term follow-up may reveal increased difference between clips and sutures with time. Further studies addressing long-term efficacy of clips are thus still needed.

The clips technique has increased in popularity, and a larger proportion of patients operated in recent years received clips compared to sutures and non-closure. Since duration of surgery falls with increased experience of the surgical team, this factor is likely to affect comparison between groups [3]. We have attempted to compensate for this using adjusted linear regression analysis, but the mean operation time is likely to overestimate the time reduction related to the technique used for mesenteric defects closure. Non-closure of the mesenteric defects was more common in the early stages of the study. Closure of the mesenteric defects became more common later on in the study. Since operation time improves with time and increase in experience, this is probably the explanation for this difference.

Finally, OP-score and bodily pain from the SF-36RAND were used as measures of patient-reported outcome. Missing data on these parameters, both at baseline and after 2 years, limits the interpretation and reliability of these outcome measures. However, it is unlikely that the missing data would cause differential bias between the study groups. Quality-oflife is an important aspect when comparing different methods of treatment and was thus included in this study, although interpretation of the results should be regarded with caution.

\section{Conclusion}

Closure of the mesenteric defects using either non-absorbable metal clips or non-absorbable running sutures is a safe and effective measure to reduce the risk for small bowel obstruction. Sutures appear slightly more effective than clips and should be considered gold standard for mesenteric defects closure.

Funding This work was supported by the grants from the Örebro Region County Council and Örebro University.

\section{Compliance with Ethical Standards}

Conflict of Interest Ingmar Näslund has received consultant fees from Baricol Bariatrics AB, Sweden. None of the remaining authors declare conflicts of interest.
Ethical Approval The study was conducted in accordance with the ethical standards of the 1964 Helsinki Declaration and its later amendments and with the approval of the regional ethics committee in Uppsala, Sweden.

Open Access This article is distributed under the terms of the Creative Commons Attribution 4.0 International License (http:// creativecommons.org/licenses/by/4.0/), which permits unrestricted use, distribution, and reproduction in any medium, provided you give appropriate credit to the original author(s) and the source, provide a link to the Creative Commons license, and indicate if changes were made.

Publisher's Note Springer Nature remains neutral with regard to jurisdictional claims in published maps and institutional affiliations.

\section{References}

1. Sjostrom L, Lindroos AK, Peltonen M, et al. Lifestyle, diabetes, and cardiovascular risk factors 10 years after bariatric surgery. $\mathrm{N}$ Engl J Med. 2004;351(26):2683-93.

2. Hutter MM, Randall S, Khuri SF, et al. Laparoscopic versus open gastric bypass for morbid obesity: a multicenter, prospective, riskadjusted analysis from the National Surgical Quality Improvement Program. Ann Surg. 2006;243(5):657-62.

3. Stenberg E, Szabo E, Agren G, et al. Early complications after laparoscopic gastric bypass surgery: results from the scandinavian obesity surgery registry. Ann Surg. 2014;260(6):1040-7.

4. Angrisani L, Santonicola A, Iovino P, et al. Bariatric surgery worldwide 2013. Obes Surg. 2015;25(10):1822-32.

5. Abasbassi M, Pottel H, Deylgat B, et al. Small bowel obstruction after antecolic antegastric laparoscopic Roux-en-Y gastric bypass without division of small bowel mesentery: a single-centre, 7-year review. Obes Surg. 2011;21(12):1822-7.

6. Higa KD, Ho T, Boone KB. Internal hernias after laparoscopic Roux-en-Y gastric bypass: incidence, treatment and prevention. Obes Surg. 2003;13(3):350-4.

7. Moore KA, Ouyang DW, Whang EE. Maternal and fetal deaths after gastric bypass surgery for morbid obesity. N Engl J Med. 2004;351(7):721-2.

8. Stenberg E, Szabo E, Ottosson J, et al. Outcomes of laparoscopic gastric bypass in a randomized clinical trial compared with a concurrent national database. Br J Surg. 2017;104(5):562-9.

9. Jacobsen H, Dalenback J, Ekelund M, et al. Tensile strength after closure of mesenteric gaps in laparoscopic gastric bypass: three techniques tested in a porcine model. Obes Surg. 2013;23(3): $320-4$.

10. Stenberg E, Szabo E, Agren G, et al. Closure of mesenteric defects in laparoscopic gastric bypass: a multicentre, randomised, parallel, open-label trial. Lancet. 2016;387(10026):1397-404.

11. Hedenbro JL, Naslund E, Boman L, et al. Formation of the Scandinavian obesity surgery registry, SOReg. Obes Surg. 2015;25(10):1893-900.

12. Olbers T, Lonroth H, Fagevik-Olsen M, et al. Laparoscopic gastric bypass: development of technique, respiratory function, and longterm outcome. Obes Surg. 2003;13(3):364-70.

13. Aghajani E, Jacobsen HJ, Nergaard BJ, et al. Internal hernia after gastric bypass: a new and simplified technique for laparoscopic primary closure of the mesenteric defects. J Gastrointest Surg. 2012;16(3):641-5.

14. Dindo D, Demartines N, Clavien PA. Classification of surgical complications: a new proposal with evaluation in a cohort of 
6336 patients and results of a survey. Ann Surg. 2004;240(2):20513.

15. Karlsson J, Taft C, Sjostrom L, et al. Psychosocial functioning in the obese before and after weight reduction: construct validity and responsiveness of the obesity-related problems scale. Int $\mathrm{J}$ Obes Relat Metab Disord. 2003;27(5):617-30.

16. Sullivan M, Karlsson J, Ware Jr JE. The Swedish SF-36 health survey-I. Evaluation of data quality, scaling assumptions, reliability and construct validity across general populations in Sweden. Soc Sci Med. 1995;41(10):1349-58.

17. Hogestol IK, Chahal-Kummen M, Eribe I, et al. Chronic abdominal pain and symptoms 5 years after gastric bypass for morbid obesity. Obes Surg. 2017;27(6):1438-45.

18. Kristensen SD, Floyd AK, Naver L, et al. Does the closure of mesenteric defects during laparoscopic gastric bypass surgery cause complications? Surg Obes Relat Dis. 2015;11(2): 459-64.

19. Brolin RE. The antiobstruction stitch in stapled Roux-en-Y enteroenterostomy. Am J Surg. 1995;169(3):355-7.

20. Aghajani E, Nergaard BJ, Leifson BG, et al. The mesenteric defects in laparoscopic Roux-en-Y gastric bypass: 5 years follow-up of non-closure versus closure using the stapler technique. Surg Endosc. 2017;31(9):3743-8.

21. Munier P, Alratrout H, Siciliano I, et al. Bidirectional Jejunojejunal anastomosis prevents early small bowel obstruction due to the kinking after closure of the mesenteric defect in the laparoscopic Roux-en-Y gastric bypass. Obes Surg. 2018;28:1838-44.
22. Watters DA, Guest GD, Tangi V, et al. Global surgery system strengthening: it is all about the right metrics. Anesth Analg. 2018;126(4):1329-39.

23. Geubbels N, Lijftogt N, Fiocco M, et al. Meta-analysis of internal herniation after gastric bypass surgery. Br J Surg. 2015;102(5): 451-60.

24. de la Cruz-Munoz N, Cabrera JC, Cuesta M, et al. Closure of mesenteric defect can lead to decrease in internal hernias after Roux-enY gastric bypass. Surg Obes Relat Dis. 2011;7(2):176-80.

25. Brolin RE, Kella VN. Impact of complete mesenteric closure on small bowel obstruction and internal mesenteric hernia after laparoscopic Roux-en-Y gastric bypass. Surg Obes Relat Dis. 2013;9(6):850-4.

26. Ahmed AR, Rickards G, Husain S, et al. Trends in internal hernia incidence after laparoscopic Roux-en-Y gastric bypass. Obes Surg. 2007;17(12):1563-6.

27. Ortega E, Morinigo R, Flores L, et al. Predictive factors of excess body weight loss 1 year after laparoscopic bariatric surgery. Surg Endosc. 2012;26(6):1744-50.

28. Musella M, Milone M, Bellini M, et al. Effect of bariatric surgery on obesity-related infertility. Surg Obes Relat Dis. 2012;8(4): 445-9.

29. Vannevel V, Jans G, Bialecka M, et al. Internal herniation in pregnancy after gastric bypass: a systematic review. Obstet Gynecol. 2016;127(6):1013-20.

30. Stuart A, Kallen K. Risk of abdominal surgery in pregnancy among women who have undergone bariatric surgery. Obstet Gynecol. 2017;129(5):887-95. 